\title{
HOW CAN CELLS SENSE THE ELASTICITY OF A SUBSTRATE? AN ANALYSIS USING A CELL TENSEGRITY MODEL
}

\author{
G. De Santis ${ }^{1, *}$, A.B. Lennon ${ }^{2}$, F. Boschetti ${ }^{3}$, B. Verhegghe ${ }^{1}$, P. Verdonck ${ }^{1}$ and P.J. Prendergast ${ }^{2}$ \\ ${ }^{1}$ Institute Biomedical Technology (IBiTech), Ghent University, Gent, Belgium \\ ${ }^{2}$ Trinity Centre for Bioengineering, School of Engineering, Trinity College, Dublin, Ireland \\ ${ }^{3}$ LaBS, Department of Structural Engineering, Politecnico di Milano, Milan \\ and IRCCS Istituto Ortopedico Galeazzi, Milan, Italy
}

\begin{abstract}
A eukaryotic cell attaches and spreads on substrates, whether it is the extracellular matrix naturally produced by the cell itself, or artificial materials, such as tissueengineered scaffolds. Attachment and spreading require the cell to apply forces in the $\mathrm{nN}$ range to the substrate via adhesion sites, and these forces are balanced by the elastic response of the substrate. This mechanical interaction is one determinant of cell morphology and, ultimately, cell phenotype. In this paper we use a finite element model of a cell, with a tensegrity structure to model the cytoskeleton of actin filaments and microtubules, to explore the way cells sense the stiffness of the substrate and thereby adapt to it. To support the computational results, an analytical 1D model is developed for comparison. We find that (i) the tensegrity hypothesis of the cytoskeleton is sufficient to explain the matrix-elasticity sensing, (ii) cell sensitivity is not constant but has a bell-shaped distribution over the physiological matrix-elasticity range, and (iii) the position of the sensitivity peak over the matrix-elasticity range depends on the cytoskeletal structure and in particular on the F-actin organisation. Our model suggests that $\mathrm{F}$-actin reorganisation observed in mesenchymal stem cells (MSCs) in response to change of matrix elasticity is a structural-remodelling process that shifts the sensitivity peak towards the new value of matrix elasticity. This finding discloses a potential regulatory role of scaffold stiffness for cell differentiation.
\end{abstract}

Keywords: Biophysical model, tensegrity, matrix elasticity, Finite Element Method, analytical model, mesenchymal stem cells.

\author{
*Address for correspondence: \\ G. De Santis \\ Institute Biomedical Technology (IBiTech) \\ Ghent University \\ De Pintelaan 185, Block B \\ BE-9000 Gent, Belgium
}

E-mail: gianluca.desantis@ugent.be
Eukaryotic cells are typically connected to extracellular matrix and/or other cells which may have very different material elasticity ranging from 0.1 to $100 \mathrm{kPa}$ (Bao and Suresh, 2003; Yeung et al., 2005). Differences in matrix elasticity cause reactions to both cellular morphology and biochemical activity (Stamenovic and Wang, 2000; Maguire et al., 2007). For example, fibroblasts cultured on compliant substrates (e.g., low-crosslinked polyacrylamide or Matrigel) adopt a small rounded shape, are without F-actin stress fibres, and present few and weak focal adhesion complexes (FACs). In contrast, on a stiff substrate (e.g., high-crosslinked polyacrylamide gels, tissue-culture plastic or glass) they flatten and spread over a 10-15-fold area (Yeung et al., 2005), present thick stress fibres (Georges and Janmey, 2005), and bind to the substrate with many strong FACs (Chen et al., 2003). Moreover, cell response to matrix elasticity is phenotype dependent and is characteristic of nervous, epithelial, endothelial, smooth muscle, bone, and cancer cells (Zhu et al., 2000; Peyton and Putnam, 2005). Using elastically-tunable matrices, Engler and collaborators have measured the response of differentiated cells to different elastic matrices (fluorescence intensity of differentiation markers) and have found a relation between the range of matrix elasticity to which the cell is sensitive and the cell phenotype. Repeating the experiment with mesenchymal stem cells (MSCs), they observed that the fluorescence intensity of differentiation markers peaked at substrate elasticity typical of each tissue type, suggesting that, unlike differentiated cells, MSCs in the first week have the potential to tune their cell-to-substrate sensitivity to the elasticity of the substrate on which they are cultured (Engler et al., 2006). More recently, embryonic stem cells have also been proven to differentiate according to the elasticity of their substrate, suggesting a primary role of matrix elasticity in cellular differentiation in the developing embryo (Evans et al., 2009). Additional observations and implications of the cell-dependent response to matrixstiffness can be found in (Discher et al., 2005; Pedersen and Swartz, 2005). A necessary condition for the different cellular reactions in response to matrices with different stiffness is that the cell is mechanosensitive to the elasticity of its matrix - one may say it "feels" the matrix stiffness (Discher et al., 2005). How this might be done by a cell is the object of this study.

The role of the cytoskeleton (CSK) in matrix elasticity sensing is supported by a number of experimental observations: (i) actin filaments (AFs) are tensed in the cell 
because they appear straight despite their short persistent length (Boal, 2002; Ingber, 2003; Kumar et al., 2006), (ii) adherent cells pull on their FACs, exerting traction forces on a passive substrate (Ingber, 2003), (iii) matrix-elasticity sensing capability is lost if the contractility of the actomyosin units is inhibited (Engler et al., 2006; Kumar et al., 2006). Topologically, the acto-myosin units are located at the FACs, which pin the AFs to the matrix. Thus, the actomyosin contraction force is transmitted by the AFs (under tension) partially to the rest of the cell (prestressed, under compression) and partially to the matrix (under traction) (Gordon and Bushnell, 1979; Sanger et al., 1984; Boal, 2002; Ingber, 2003; Kumar et al., 2006). It is also known that microtubules (MTs) are compressed in the cell because they appear highly curved despite their long persistent length (Brangwynne et al., 2006). All these considerations agree with the hypothesis of tensegrity structure of the CSK in which the MTs are compression-supporting elements which, together with the matrix, balance the tension raised from the acto-myosin contraction.

In this study, tensegrity organisation of the CSK is assumed and included into a biophysical model of an adherent cell, in order to investigate whether the tensegrity hypothesis is sufficient to explain the mechanical principle of matrix-elasticity sensing. Such a model is then applied to explore the way a cell could adapt to a specific substrate, by regulating acto-myosin contraction and AF bundling. A more intuitive analytical model of the adherent cell has been designed ad hoc to validate the results of the computational model. Remarkably, our model is topologically static, and rearrangements of the cytoskeletal components, such as formation of new FACs and stress fibres, is not considered.

\section{Methods}

\section{Tensegrity-based computational model}

A computational model of an adherent eukaryotic cell has been developed by including the cellular components likely to be structurally significant: nucleus, cytoplasm and membrane (modelled as linear elastic continua (Lim et al., 2006)), and the CSK (modelled as a tensegrity structure (Wendling et al., 1999; McGarry and Prendergast, 2004)). The substrate on which the cell is adhered has also been modelled as a linear elastic isotropic continuum (Fig. 1). The material properties of the subcellular components have been taken from literature: cytoplasm and nucleus were assigned Young's modulus (E) of $100 \mathrm{~Pa}$ and 400 $\mathrm{Pa}$, respectively, and Poisson's ratio (v) 0.37 (Maniotis et al., 1997; Guilak et al., 2000; Deguchi et al., 2005); cell membrane was modelled with a thickness of $6 \mathrm{~nm}, \mathrm{E}=1$ $\mathrm{kPa}$ and $v=0.3$ (Kamm et al., 2000); in the CSK, crosssectional area of $20 \mathrm{~nm}^{2}$ and $200 \mathrm{~nm}^{2}$ have been assigned to AFs and MTs, with $\mathrm{E}=1 \mathrm{GPa}$ (Gardel et al., 2008).

The geometry of the cell has been developed and meshed in pyFormex, a python-based open-source software, used to create and to handle large geometrical models (Web ref. 1). A parabolic profile has been revolved around an axis reproducing a cell in a spread configuration (radius of the bottom side $18 \mu \mathrm{m}$ and height $6 \mu \mathrm{m}$ ). The nucleus has been modelled as an ellipsoid (radii 4 and 2.5 $\mu \mathrm{m})$ with the centre positioned $4.5 \mu \mathrm{m}$ from the substrate. The remaining space has been filled with cytoplasm and covered with a surface representing the cell membrane. Only MTs and AFs have been included in the CSK, as parts of a tensegrity structure with 12 compression-only struts and 36 tension-only struts (cables). Struts and cables of the tensegrity structure idealise the structural role of AFs and MTs, respectively, and were connected to each other by translation-only constraints (i.e., pin-joints). For the simulation, the compression-only behaviour of MTs has not been explicitly set but has been verified a posteriori. Indeed, in the cell MTs could also support tensional forces even if they appear compressed and highly curved in living cells. Intermediate filaments have not been explicitly considered in the CSK because their supporting role has been reported to be relevant for high cellular deformations only (strain higher than $20 \%$ ) (Janmey et al., 1991; Wang and Stamenovic, 2000). The cell model has been attached to a flat substrate, as shown in Fig. 1D.

Cytoplasm, nucleus and substrate were meshed with 8 -node hexahedral elements and the outer faces of the elements of the cytoplasm (quadrilaterals) were used as shell elements of the cell membrane. The resulting mesh of 15,132 elements was imported into Abaqus for the finite element analysis. The 24 vertices of the tensegrity structure were constrained to the closest nodes of the cell membrane by pin joint connectors representing cell mechanoreceptors, 'receptor' sites where actin filaments cluster at adhesion complexes in adherent cells (Wang and Stamenovic, 2000). The nodes at the bottom face of the substrate were constrained in all degrees of freedoms, while on the top face relative movements between cell and substrate were allowed, with the exception of the four bottom vertices of the CSK that were pinned on the substrate. These locations represent the FACs which are known to mechanically couple the cell to the substrate; i.e. contact between cell and substrate occurs only at the FAC sites but not in the areas between them (Chicurel et al., 1998; Chen et al., 2003) (Fig. 1D). In order to avoid artefacts due to finite dimensions of the substrate model, the radius of the substrate was chosen twice as long as the distance of the FACs from the centre of the cell-substrate interface (Sen et al., 2009).

The Finite Element Method was used to evaluate the effect of acto-myosin contraction on the cell-substrate force balance. In a real cell, acto-myosin units are connected to the AFs and act as tension-generators. This pre-tension was achieved in the simulated cell by defining the resting length of the tensegrity cable (representing the AFs) as a function of a state variable $\mathrm{P}$, according to the following linear equation:

$$
L=\alpha L_{0}\left(P-P_{0}\right)+L_{0}
$$

An isolated AF having a resting length $\mathrm{L}_{0}$ at an initial value of the state variable $\mathrm{P}_{0}$ shrinks to a length $\mathrm{L}_{\mathrm{f}}$ after the state variable drops to $\mathrm{P}_{\mathrm{f}}$, with $\alpha$ being the coefficient of the linear relation between $P$ and L. Because in our model AFs are not isolated but constrained at their endings to the cell membrane, shrinking of AFs generates tensional 
A

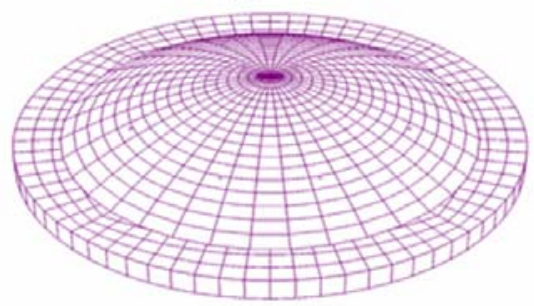

C

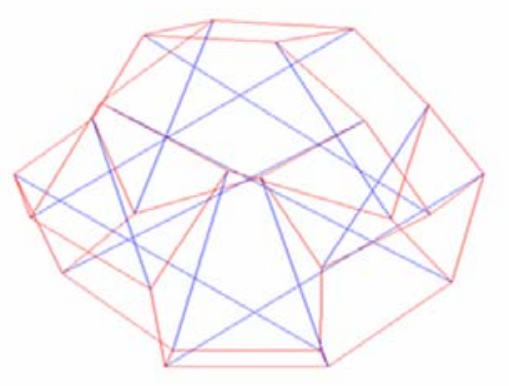

B

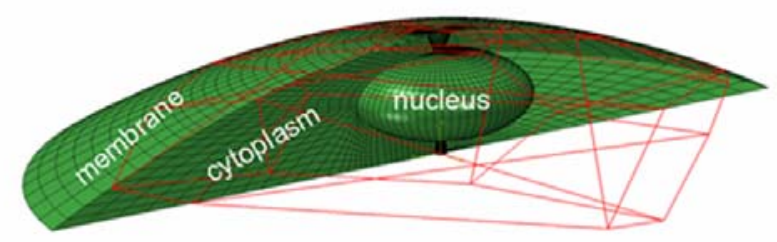

D

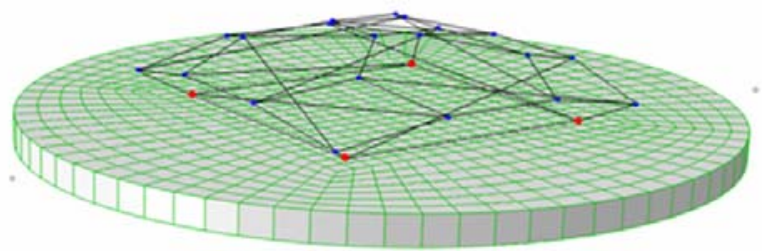

Fig. 1. Finite element model of an adherent cell. (A) Cell adhered on a substrate, (B) Cut view of the cell, (C) Unscaled tensegrity structure used for the CSK with red AFs and blue MTs, (D) The CSK is connected to the substrate by four FACs.

forces, which in turn are balanced by compressive forces in the rest of the cell and traction on the surface of the substrate that is connected to the cell by the FACs. The force-balance resulting from cell contraction has been simulated for values of substrate elasticity spread over six orders of magnitude - from 0.1 to $100,000 \mathrm{kPa}$. For this first analysis, a stiffness corresponding to a bundle of 16 AFs was assigned to each cable of the tensegrity structure, representing stress fibres (SFs). The cell contraction modelled as cable pre-strain has been obtained by reducing the resting length of each cable by $1 \%$ (Eq. 1). Mesh independence of the results has been assessed by doubling the number of elements as well as using different element types (tetrahedral / triangular elements).

Cytoskeletal adaptation by changing cell contractility or the thickness of SFs (by increasing the number of AFs in each bundle) has been investigated by performing a parametric analysis. Cell contractility has been evaluated by reducing the resting length of each cable by $0.5,2$ and $8 \%$ while AF assembly has been evaluated by increasing cable thickness to represent bundling of up to $128 \mathrm{AFs}$ (thick SFs) in each cable of the tensegrity structure. The parametric analysis of CSK structure corresponds to modelling the CSK of different cell phenotypes, from nervous cells, having single AFs without striations / stress fibres, to myocytes and osteoblasts having AFs bundled in differently thick SFs (Gardel et al., 2004). The average displacement of FACs and the average force carried by the MTs were calculated using finite element analyses. The derivative of the MT force with respect to the substrate elasticity was chosen as a measure of cell sensitivity.

\section{Analytical spring model of cytoskeleton and substrate}

The CSK was further simplified into a lumped parameter model of two springs in parallel, representing MTs and AFs (by definition this is still a tensegrity structure). By including the matrix (or substrate SS) as a third component the simplest model of cell-substrate force balance was generated as shown in Fig. 2A.

Each spring was characterised by a linear forcedisplacement relation with spring constant $\mathrm{k}$. In case of AFs and MTs, the value of $\mathrm{k}$ could be derived from the constitutive equation of a single strut element used in the tensegrity model, as

$$
\begin{aligned}
& F=A \cdot E \cdot \frac{\left(L-L_{0}\right)}{L_{0}} \\
& F=k \Delta L=\frac{A E}{L_{0}}\left(L-L_{0}\right)
\end{aligned}
$$

where A was the cross-section, E was the Young Modulus, $\mathrm{L}$ was the actual length and $\mathrm{L}_{0}$ was the rest length. A different spring constant $\mathrm{K}$ was used instead of $\mathrm{k}$, in order to have a constant independent of the rest length (See note).

$$
K=A \cdot E
$$

The rest length of MTs and substrate was constant while the rest length of AFs depended on the prestress $\mathrm{P}$, according to the following equation

$$
L_{A F R}=(1+P) \cdot L_{0}
$$

where $\mathrm{L}_{0}$ is the rest length of AFs when $\mathrm{P}=0$. 


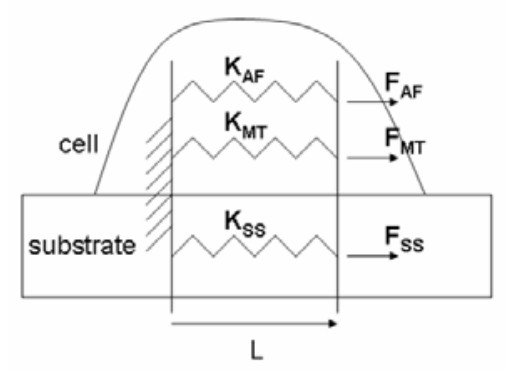

A

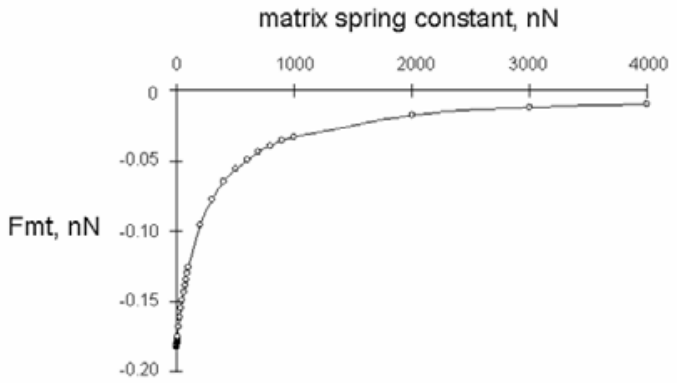

B
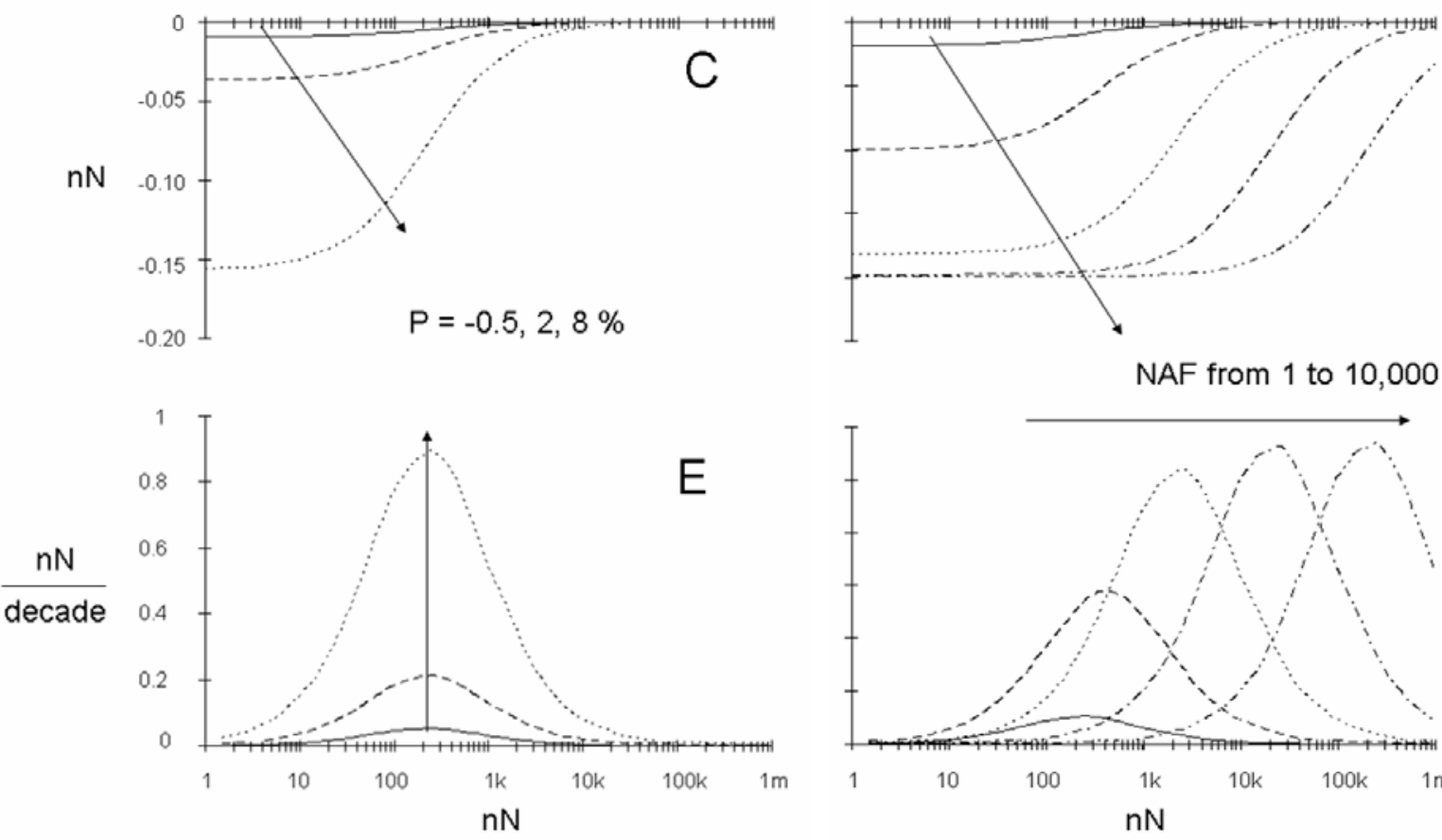

$\mathrm{D}$

Fig. 2. Cell-substrate lumped parameter model. (A) Springs representing CSK and substrate, (B) Portion of hyperbola obtained with -0.01 of prestress $(\mathrm{P})$ and material properties of a single AF and a single MT, (C) Effect of increasing the prestress on $\mathrm{F}_{\mathrm{MT}}$ and $(\mathrm{E})$ on the derivative of $\mathrm{F}_{\mathrm{MT}}$, (D) Effect of increasing the number of AFs in a bundle on $\mathrm{F}_{\mathrm{MT}}$ and (F) on the derivative of FMT, Bundling of AFs appears to magnify the peak compression force in the MTs but this effect extinguishes for stiffer AF networks (if $\mathrm{K}_{\mathrm{AF}}>>\mathrm{K}_{\mathrm{MT}}$ the peak $\mathrm{F}_{\mathrm{MT}} \sim \mathrm{K}_{\mathrm{MT}}{ }^{*} \mathrm{P}$, from Eq. 8).

In order to reduce the number of parameters, the rest lengths of MTs and substrate was taken equal to $\mathrm{L}_{0}$ without losing generality, thus reducing the three spring equations to

$$
\begin{gathered}
F_{A F}=K_{A F} \cdot\left(L-L_{A F R}\right) / L_{A F R} \\
F_{M T}=K_{M T} \cdot\left(L-L_{0}\right) / L_{0} \\
F_{S S}=K_{S S} \cdot\left(L-L_{0}\right) / L_{0}
\end{gathered}
$$

The equilibrium condition of the three springs in parallel was given by

$$
F_{A F}+F_{M T}+F_{S S}=0
$$

Combining Eq. 4 and Eqs. 5 into Eq. 6, displacement and force in the MTs could be calculated as

$$
L-L_{0}=\frac{L_{0} \cdot K_{A F} \cdot P}{K_{A F}+(1+P) \cdot\left(K_{M T}+K_{S S}\right)}
$$

$$
F_{M T}=\frac{K_{M T} \cdot K_{A F} \cdot P}{K_{A F}+(1+P) \cdot\left(K_{M T}+K_{S S}\right)}
$$

As in the tensegrity-based model, the cell-substrate force balance was simulated by changing the substrate stiffness (Kss), and the cytoskeletal adaptation by changing the contractility (i.e. the prestress $\mathrm{P}$ of the $\mathrm{AFs}$ ) or the thickness of SFs (i.e., the number of AFs).

Note: In this model the conventional force-displacement spring constant $\mathrm{k}$ depends on the prestress because the rest length of AFs is a function of the prestress (Eq. 1). Therefore, a different prestress-independent constant $\mathrm{K}$ (force-strain constant) is introduced $(\mathrm{K}=\mathrm{k} \cdot \mathrm{L} 0=\mathrm{A} \cdot \mathrm{E})$.

\section{Results}

\section{Tensegrity-based computational model}

The computational cell model pulls on its FACs producing on the surface of the substrate a traction directed towards the centre of the cell-substrate interface, generating a 

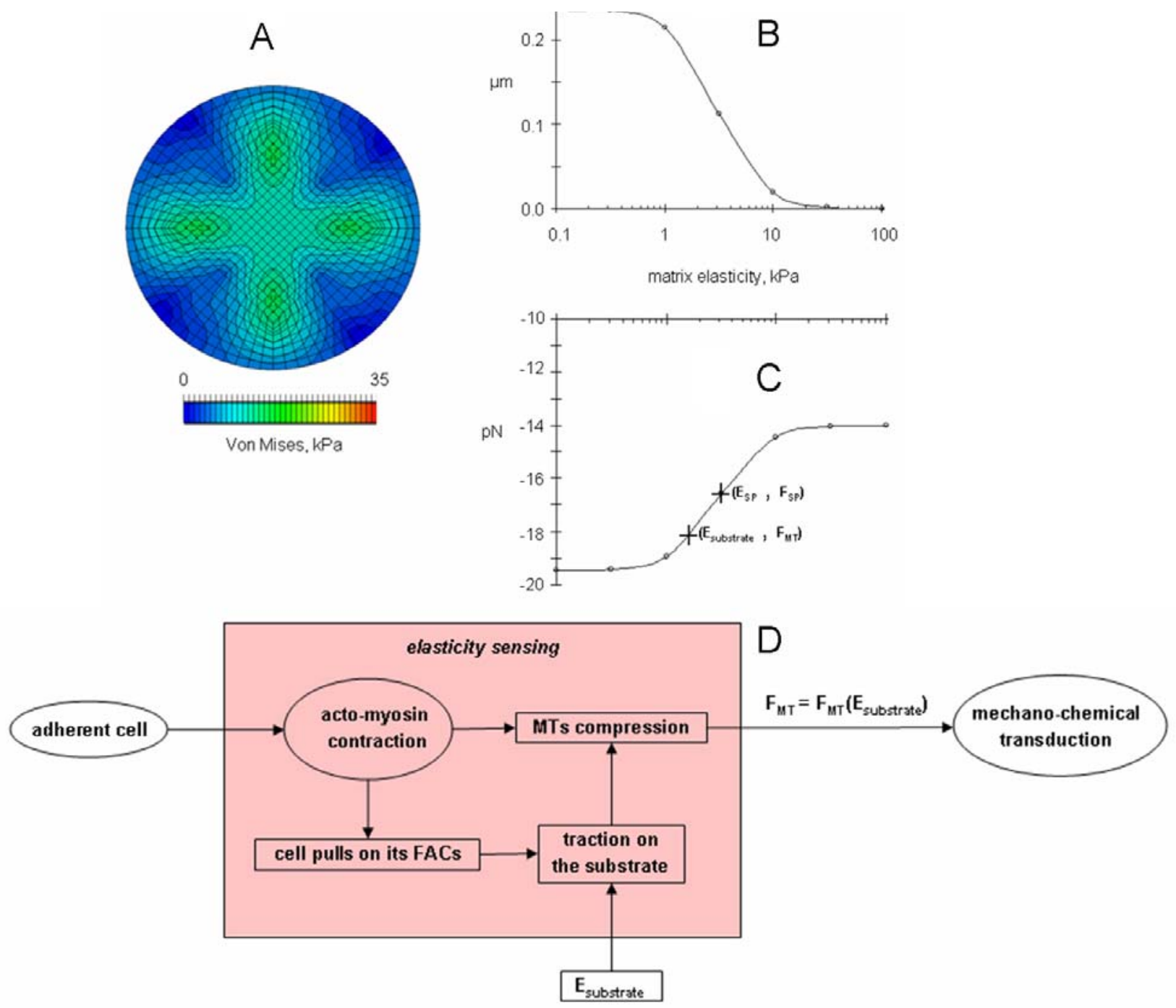

Fig. 3. Cell senses the substrate stiffness by pulling on it. (A) Plot of the Von Mises stress on the top surface of the substrate, (B) Average displacement of the four FACs and (C) average longitudinal force acting on the MTs in response to $1 \%$ pre-strain applied to SFs. Matrix elasticity is represented on logarithmic axis, (D) When a cell adheres on a substrate with elasticity $\mathrm{E}_{\text {substrate }}$ a force $\mathrm{F}_{\mathrm{MT}}$ acts on the MTs. The cell has a particular value of $\mathrm{E}_{\text {substrate }}$ to which it is maximally sensitive $\left(\mathrm{E}_{\mathrm{SP}}=\right.$ sensitivity peak elasticity): maximal variation of MT force - or FAC displacement - in response to $\mathrm{E}_{\text {substrate }}$ variation. The value of $\mathrm{E}_{\text {substrate }}$ determines the force $\mathrm{F}_{\mathrm{MT}}$. This force can initiate different mechano-chemical events. The coloured box contains this proposed principle of mechanosensation.

stress distribution in the substrate with a peak stress under each FAC. Edge effects due to limited dimension of the substrate model were judged negligible as the stress values calculated at the boundary of the substrate were predicted to be close to zero (Fig. 3A). Stiffer substrates restrain the cell more resulting in a lower displacement of the FAC. The FAC displacement decreases over the substrate elasticity (in logarithmic scale) with a sigmoid-like fashion (Fig. 3B). As the substrate becomes stiffer, it exerts a stronger resistance against the FAC displacement, and thus carries a greater portion of the compression needed to balance the actin-filament tension. Consequently, the intensity of compression forces in the MTs gradually decreases as the substrate stiffens (Fig. 3C). The dependence of MT force on substrate elasticity provides a possible mechanical principle underlying a cell's capability for matrix-elasticity sensing (Fig. 3D). A mechanical basis for mechanosensitivity has been recently considered, suggesting a relevant role of FACs and SFs (Nicolas et al., 2004; Deshpande et al., 2007; Walcott and Sun, 2010; Zemel et al., 2010). In our model, the relation between MT force and substrate elasticity is described by a sigmoid-like curve which demonstrates that the cell-to-substrate-elasticity sensitivity is not constant but depends on the elasticity value (cell sensitivity is the MTforce variation in response to 10 -fold substrate-elasticity variation in a logarithmic scale). Cell sensitivity was predicted to have a bell-shaped distribution, which shows that a cell can have one single peak of sensitivity for a particular value of substrate elasticity. The more the matrix elasticity differs from this peak value, the more the cell loses sensitivity to matrix-elasticity variations (Fig. 4C,F).

Stronger cell contraction due to greater tension generated by the acto-myosin motors increases both the FAC displacement (Fig. 4A) and the compression in the 

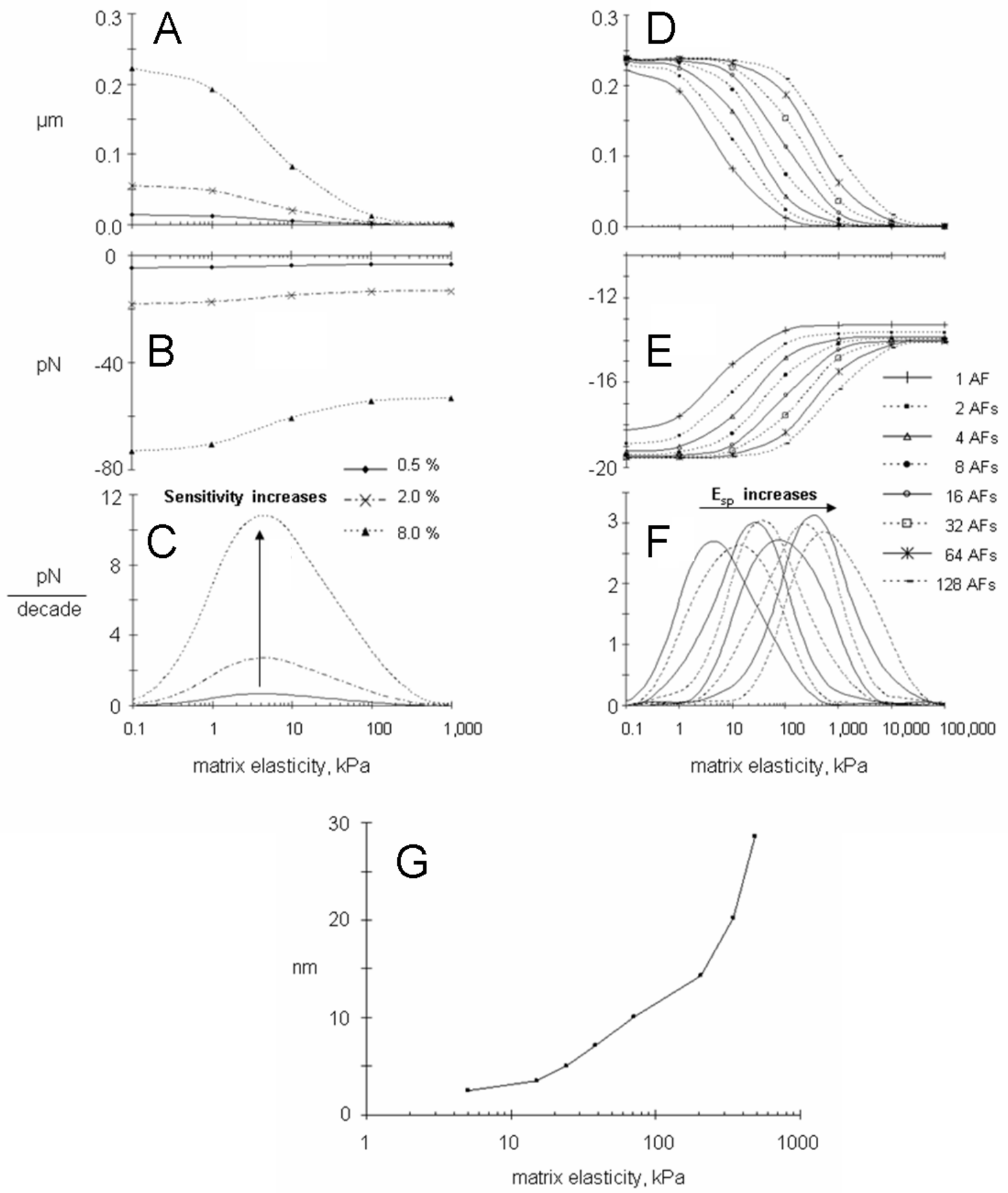

Fig. 4. Effects of contractility level and AF bundling on cell-to-matrix sensitivity. Increasing the cable pre-strain (contractility) from $0.5 \%$ to $8 \%$ produces stronger variations of FAC displacement (A) and MT force (B) resulting in higher cell to substrate sensitivity (C, derivative of the curves in B). Bundling of AFs (increasingly thicker SFs) produces minor effect on FAC displacement (D), MT force (E) and cell sensitivity values (F), but translates the Esp (sensitivity peak elasticity) towards stiffer substrates, making the cell sensitive to different types of substrates. An adherent cell requires thicker SFs to feel the elasticity of stiffer matrices (the effect of bundling up to 128 AFs per $\mathrm{SF}$ is represented in $\mathrm{G}$, with $\mathrm{SF}$ radius on y-axis).

MTs (Fig. 4B), enhancing the value of cell sensitivity but without altering the value of matrix elasticity where the sensitivity peak is located (Fig. 4C). A different effect is produced by AF bundling. As SFs become thicker the sigmoid-like curve of sensitivity translates from left to right (Fig. 4D,E), moving the peak of sensitivity from low elasticity value to higher elasticity values of the substrate (Fig. 4F). Thus, modulating the elasticity value corresponding to the sensitivity peak is only possible by AF bundling and not by changing the cell contractility. Assuming that an adherent cell adapts its CSK in order to maximize its sensitivity, then AF bundling is a possible 
strategy of adaptation, as the size of the SFs determines the value of substrate elasticity to which the cell has the highest sensitivity (Fig. 4G).

\section{Analytical spring model of cytoskeleton and substrate}

The analytical model predicts a displacement directly proportional to the $\mathrm{F}_{\mathrm{MT}}$ by a constant $\left(\mathrm{L}_{0} / \mathrm{K}_{\mathrm{MT}}\right)$. According to the data chosen for the tensegrity model $\left(\mathrm{E}_{\mathrm{AF}}=\mathrm{E}_{\mathrm{MT}}=1\right.$ $\mathrm{GPa}, \mathrm{A}_{\mathrm{AF}}=20 \mathrm{~nm}^{2}, \mathrm{~A}_{\mathrm{MT}}=200 \mathrm{~nm}^{2}$ ), the spring constants for AFs and MTs are equal to 20 and $200 \mathrm{nN}$, respectively. The relation between $\mathrm{F}_{\mathrm{MT}}$ and $\mathrm{K}_{\mathrm{SS}}$ (Eq. 8) is a portion of hyperbola (also known as homographic function) as shown in Fig. 2B. On a logarithmic scale, necessary to visualize the $\mathrm{F}_{\mathrm{MT}}$ curve on several decades of substrate spring constant, the $\mathrm{F}_{\mathrm{MT}}$ appears as sigmoid, with highest cell-to-substrate sensitivity (highest derivative of $\mathrm{F}_{\mathrm{MT}}$ ) on one specific substrate stiffness. Altering parametrically the prestress $\mathrm{P}$ of the AFs and the number of AFs $\left(\mathrm{N}_{\mathrm{AF}}\right)$ in the spring element $\left(\mathrm{K}_{\mathrm{NAF}}=\mathrm{K}_{\mathrm{AF}} \cdot \mathrm{N}_{\mathrm{AF}}\right)$, the $\mathrm{F}_{\mathrm{MT}}$ curve changes as shown in Fig.2 C,D.

The analytical model confirms the outcomes of the FE model, reflecting a sigmoid-like fashion for both $\mathrm{F}_{\mathrm{MT}}$ and FAC displacement and a bell-shape sensitivity diagram. Moreover, a prestress variation (acto-myosin contraction) only affects the $\mathrm{F}_{\mathrm{MT}}$ magnitude (Fig.2 C,E) while AF bundling moves the sensitivity peak towards stiffer substrates (Fig.2 D,F). Remarkably, an important feature of the FE model is lost in the spring model: on a rigid substrate (zero FACs displacement) MTs are unloaded while in the 3D tensegrity model MTs are still under compression.

\section{Discussion}

This computational study investigates the mechanical principle underlying a cell's capability for matrix-elasticity sensing by considering only cellular contractility as a possible mechanical load. Initially, tensional loads are actively generated by continuous acto-myosin contraction and are carried by the AFs, which contain these motor proteins. The generated tension is balanced by compression that is shared by the cellular continua (cytoplasm, nucleus and cell membrane), the MTs, and the traction exerted by the cell on its substrate through its FACs. Despite its simplicity, the proposed model predicts a one-toone relationship between substrate elasticity and MT compression forces. This relation is theoretically sufficient to provide the cell with the capability of matrix-elasticity sensing. Indeed a specific level of MT compression can initiate several cellular biochemical activities by (i) gating mechano-sensitive ion channels, (ii) altering intracellular CSK-related biochemistry (e.g. enzymatic activity) and gene expression (Chicurel et al., 1998), and (iii) exerting forces on the nuclear scaffold through discrete mechanical paths (Maniotis et al., 1997).

The relation between substrate elasticity and MT forces is non-linear, represented by a sigmoid curve over a logarithmic axis of the substrate elasticity. Consequently, cell to substrate-elasticity sensitivity (i.e. the derivative of the sigmoid) is a bell-shaped profile meaning that a cell has a maximal sensitivity for a specific-elasticity value (sensitivity peak) and that this capability is gradually lost going far from this value. With respect to the elasticitydependent cell sensitivity just found, two possible CSK remodelling strategies have been investigated by means of parametric analysis (contractility and AF assembling). Results indicate that contractility is required and determines the level of cell sensitivity but does not influence the range of matrix-elasticity to which the cell is sensitive. Only by AF bundling was the cell model able to regulate its sensitivity to a specific substrate, making AF bundling a strong candidate for a regulatory mechanism for cell-tosubstrate induced structural adaptation.

To validate our analysis, an analytical model of three springs in parallel was developed. Despite its simplicity, the analytical model predicts a similar force balance between CSK and substrate as obtained with the finite element model, with a microtubular compression force over the substrate-stiffness range described by a sigmoid-like curve. Enhancing the contractility does affect the magnitude of the compression force in the MTs but as a purely scaling effect on the sigmoid-like curve. Conversely, bundling of AFs changes the cell sensitivity, tuning the cell to stiffer substrates.

The selective nature of cell sensitivity predicted by our model has been recently demonstrated in vitro. Yeung and collaborators (Yeung et al., 2005) report that there is an elasticity range in which small variations of matrix-elasticity produce huge variations of some cellular parameters (spreading size, stress fibre content, and amount of adhesion receptors) and that this range is phenotype specific. Outside this range even huge variations of matrixelasticity result in small or no variations of the same parameters (Yeung et al., 2005). Engler and collaborators measured the fluorescence intensity of neuro-, myo- and osteo- differentiation markers on differentiated cells belonging to neuro-, myo- and osteo-phenotypes and found distributions of these markers very similar to the bell-shaped curves predicted by our model. Furthermore, they observed that low $(0.1-1 \mathrm{kPa})$, medium $(8-17$ $\mathrm{kPa})$ and high $(25-40 \mathrm{kPa})$ values of substrate-elasticity caused lineage specification of MSCs in the first week; thus they classified these substrates as neuro-, myo- and osteogenic, respectively, and showed that this specification was inhibited by administering blebbistatin, which is known to block the acto-myosin motors responsible for cell contractility. Diffuse AF networks appeared in MSCs cultured on low-stiffness matrix (neurogenic) while striations and stress fibres appeared if MSCs were cultured on medium- (myogenic) and high- (osteogenic) stiffness matrices, respectively (Engler et al., 2006). Taken together, these observations corroborate our model a posteriori and motivate its use to explain CSK related adaptation processes.

Based on the analysis of our model, we predict that not only blockage of the acto-myosin contraction but also disruption of MTs (e.g., by colchicine (Wang et al., 2001)) would inhibit the cell's ability to feel its matrix, thus reducing the influence of the microenvironment stiffness on the lineage specification and raising the influence of other stimuli. However, the role of MTs may be only transferring 

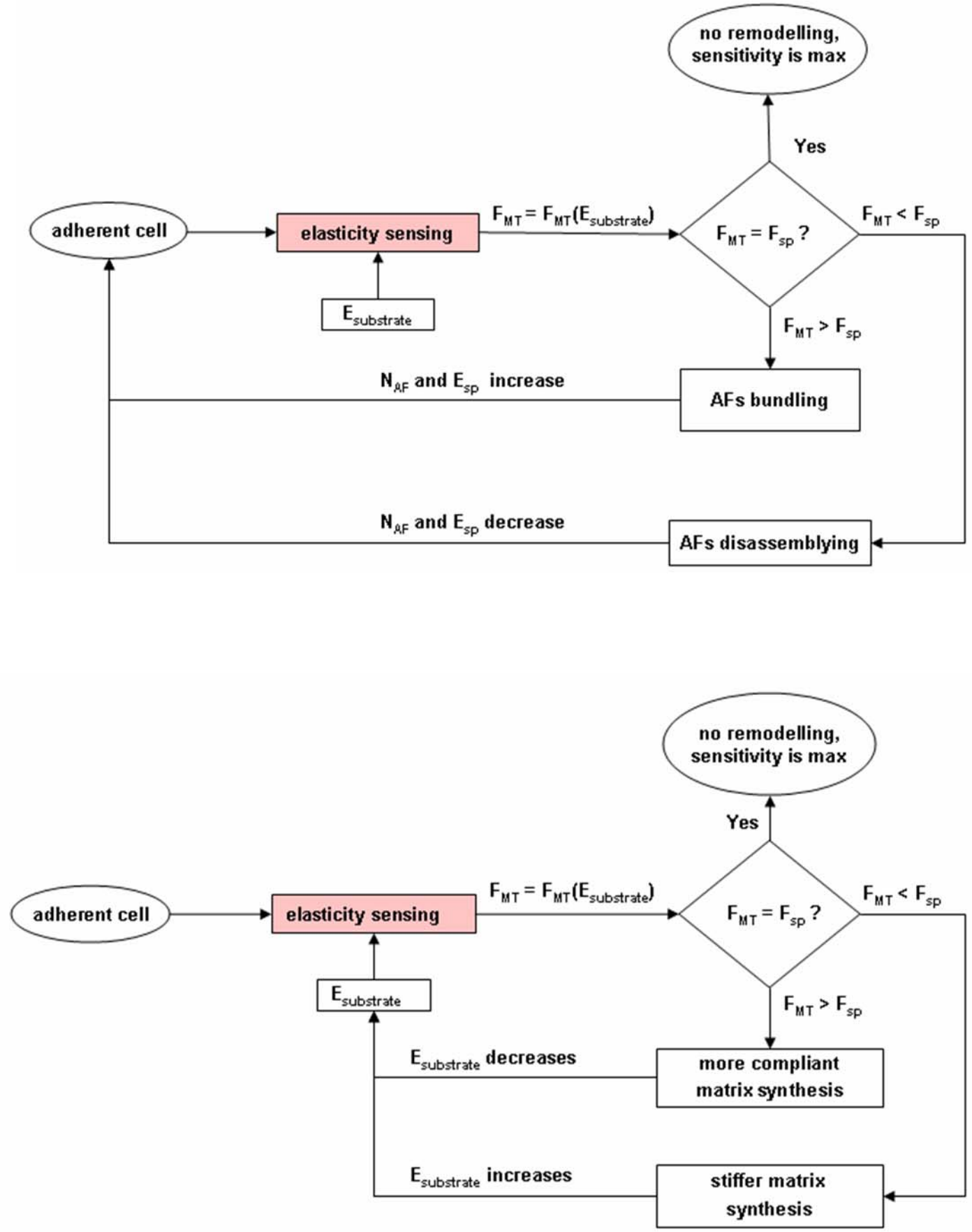

Fig. 5. Adaptation to matrix elasticity suggested from the model. (Top) Adaptation to matrix elasticity by MSCs. The force acting on the MT represents the key of substrate stiffness $\left(\mathrm{E}_{\text {substrate }}\right)$ mechanotransduction. A particular CSK configuration has a specific value of matrix elasticity $\left(\mathrm{E}_{\mathrm{SP}}\right)$ to which the sensitivity is maximal. In this particular case the MT force $\left(\mathrm{F}_{\mathrm{MT}}\right)$ is equal to $\mathrm{F}_{\mathrm{SP}}$ and there is no remodelling occurring. If $\mathrm{F}_{\mathrm{MT}}$ is higher than $\mathrm{F}_{\mathrm{SP}}$, abs $\left(\mathrm{F}_{\mathrm{MT}}\right)$ $<\operatorname{abs}\left(\mathrm{F}_{\mathrm{SP}}\right)$, the matrix is too stiff for the cell and consequently the cell starts reinforcing its SFs, by increasing the number of AFs in each bundle. Vice versa if $\mathrm{F}_{\mathrm{MT}}$ is lower than $\mathrm{F}_{\mathrm{SP}}$, abs $\left(\mathrm{F}_{\mathrm{MT}}\right)>\operatorname{abs}\left(\mathrm{F}_{\mathrm{SP}}\right)$, the matrix is too compliant and the cell starts dismantling its SFs, by decreasing the number of AFs in each bundle $\left(\mathrm{N}_{\mathrm{AF}}\right.$, Fig. $4 \mathrm{~F}$, i.e., changing the radius of each SF, Fig. 4G). Thanks to this CSK adaptation, the $\mathrm{E}_{\mathrm{SP}}$ translates towards a value closer to the current matrix elasticity $\left(\mathrm{E}_{\text {substrate }}\right)$. (Bottom) Adaptation to matrix elasticity through microenvironment remodelling. Some cells can adapt to their matrix by altering the matrix around them. Synthesis and secretion of extracellular matrix with stiffness closer to $\mathrm{E}_{\mathrm{SP}}$ allows the cell to maximize its sensitivity to its matrix without remodelling its CSK. If MT force $\left(\mathrm{F}_{\mathrm{MT}}\right)$ is equal to $\mathrm{F}_{\mathrm{SP}}$ then there is no remodelling occurring. If $\mathrm{F}_{\mathrm{MT}}$ is higher than $\mathrm{F}_{\mathrm{SP}}, \operatorname{abs}\left(\mathrm{F}_{\mathrm{MT}}\right)<\operatorname{abs}\left(\mathrm{F}_{\mathrm{SP}}\right)$, the matrix is too stiff for the cell and consequently the cell starts secreting a more compliant matrix, whereas, if $\mathrm{F}_{\mathrm{MT}}$ is lower than $\mathrm{F}_{\mathrm{SP}}, \operatorname{abs}\left(\mathrm{F}_{\mathrm{MT}}\right)>\operatorname{abs}\left(\mathrm{F}_{\mathrm{SP}}\right)$, the matrix is too compliant and the cell starts secreting a stiffer matrix. As a result, the newly secreted matrix will alter the cell microenvironment, moving the value of $\mathrm{E}_{\text {substrate }}$ towards the ESP, as shown in Fig. 3C. The elasticity sensing (coloured box) represents the coloured box in Fig. 3D. 
compression to the sensing apparatus and their disruption may not totally unlink the transmission of compression to the "sensor", which could be delivered through other components, e.g. fluid pressure in the cytosol.

Our parametrical analyses demonstrate that AF assembly is the only parameter capable of regulating cell sensitivity to substrate elasticity in our model. Consequently, the rule governing CSK remodelling in MSCs cultured on specific elasticity-characterised substrates can be explained as a maximisation process of MSC sensitivity to their substrate. Because AF bundling translates the peak of the bell-shaped curves over the substrate-elasticity axis, the AF bundling (or dismantling) observed in vitro represents a mechanical tuning of an adherent cell towards the elasticity value of its substrate (Fig. 5, top).

Maximisation of cell sensitivity to the elasticity of its substrate can also occur in another way. A cell not capable of CSK remodelling (e.g., adult differentiated cells) can adapt its substrate towards its own elasticity value of sensitivity peak. Some adult differentiated cells are capable of synthesising and secreting matrix (e.g., collagen) creating a new microenvironment (with a new elasticity level) around them (Kong et al., 2005). Such an adaptation mechanism without CSK remodelling is also explained by our model (Fig. 5, bottom).

Despite its simplicity, our biophysical model is capable of capturing the principle of matrix-elasticity sensing. To verify that such a capability is not related to the tensegrity configuration chosen, we have repeated the virtual testing with a different tensegrity structure for the CSK (6 struts, 24 cables and 3 FACs) and we have obtained curves qualitatively similar to those in Fig. 4E. However, our model neglects the effect of the up- or down-regulation of FACs on the elasticity sensing, which has been reported to affect the complementary load balance between AFs, MTs and substrate (Buxbaum and Heidemann, 1988). As anticipated in the Introduction, a cell on a stiffer substrate tends to increase the number of FACs, thus transferring loads more effectively to the substrate and reducing the loads acting on the MTs. This extra reduction of MT compression is likely to induce extra formation of stress fibres, which has the double effect of restoring the physiological MT compression (Fig. 4E) and shifting the sensitivity peak (Fig. 4F) towards the new substrate stiffness.

Living cells are in a dynamical condition with formation and degradation of the CSK. For example, FACs move under load which may be externally applied or even internally generated (like in adherent cells). The choice of neglecting CSK dynamics in our model derives from the limited knowledge of the bio-mechanical cell adaptation mechanisms which, in reality, may play a role in matrix stiffness mechano-sensation (Nicolas et al., 2004; Besser and Safran, 2006; Biton and Safran, 2010; Chan and Odde, 2008). Recently, a first attempt to mimic a biologically realistic architecture of the CSK has been reported: a form-finding model in which the structuration of a tensegrity-based CSK during cell adhesion and spreading was reproduced by means of MT growth from a centrosome towards the membrane receptors (Maurin et al., 2008). Despite the assumptions needed to overcome the lack of biological knowledge (e.g. rate of the MT growth) and its limitation (e.g., AF polymerisation was neglected), such a form-finding model could be included in our mechanosensing model, enriching the virtual cell adaptation with the dynamic formation of FACs.

\section{Conclusion}

The cell-substrate biophysical model proposed in this paper relies on current biological cell knowledge and successfully captures the mechano-sensing capability of an adherent cell on a passive substrate. It suggests a possible regulatory mechanism for MSC and adult differentiated cells adaptation to elastically-tunable substrates and demonstrates that this adaptation can lead the cell towards the maximal sensitivity to the elasticity of its matrix. Including realistic form-finding algorithms into our 'mechano-sensing' model would allow virtual-mimicking of cell processes such as adhesion, spreading and migration and could provide rational recommendations to optimize cellular response in tissue-engineering applications (e.g., scaffolds) for therapeutic use.

\section{Acknowledgments}

We gratefully acknowledge Bart Vandenbroucke for his contribution to the study.

\section{References}

Bao G, Suresh S (2003) Cell and molecular mechanics of biological materials. Nat Mater 2: 715-725.

Besser A, Safran SA (2006) Force-induced adsorption and anisotropic growth of focal adhesions. Biophys J 90: 3469-3484.

Biton YY, Safran SA (2010) Theory of the mechanical response of focal adhesions to shear flow. J Phys Condens Matter 22: 194111.

Boal DH (2002) Mechanics of the Cell. Cambridge University Press, Cambridge.

Brangwynne CP, MacKintosh FC, Kumar S, Geisse NA, Talbot J, Mahadevan L, Parker KK, Ingber DE, Weitz DA (2006) Microtubules can bear enhanced compressive loads in living cells because of lateral reinforcement. J Cell Biol 173: 733-741.

Buxbaum RE, Heidemann SR (1988) A thermodynamic model for force integration and microtubule assembly during axonal elongation. J Theor Biol 134: 379-390.

Chan CE, Odde DJ (2008) Traction dynamics of filopodia on compliant substrates. Science 322: 1687-1691.

Chen CS, Alonso JL, Ostuni E, Whitesides GM, Ingber DE (2003) Cell shape provides global control of focal adhesion assembly. Biochem Biophys Res Commun 307: 355-361.

Chicurel ME, Chen CS, Ingber DE (1998) Cellular control lies in the balance of forces. Curr Opin Cell Biol 10: $232-239$. 
Deguchi S, Maeda K, Ohashi T, Sato M (2005) Flowinduced hardening of endothelial nucleus as an intracellular stress-bearing organelle. J Biomech 38: 1751-1759.

Deshpande VS, McMeeking RM, Evans AG (2007) A model for the contractility of the cytoskeleton including the effects of stress-fibre formation and dissociation. Proc Roy Soc A Math Phys EngSci 463: 787-815.

Discher DE, Janmey P, Wang YL (2005) Tissue cells feel and respond to the stiffness of their substrate. Science 310: 1139-1143.

Engler AJ, Sen S, Sweeney HL, Discher DE (2006) Matrix elasticity directs stem cell lineage specification. Cell 126: 677-689.

Evans N, Minelli C, Gentleman E, LaPointe V, Patankar SN, Kallivretaki M, Chen X, Roberts CJ, Stevens MM (2009) Substrate stiffness affects early differentiation events in embryonic stem cells. Eur Cells Mater 18: 1-14.

Gardel ML, Kasza KE, Brangwynne CP, Liu JY, Weitz DA (2008) Mechanical response of cytoskeletal networks. Biophys Tools Biol, Vol 2: In Vivo Techn 89: 487-519.

Gardel ML, Shin JH, MacKintosh FC, Mahadevan L, Matsudaira P, Weitz DA (2004) Elastic behavior of cross-linked and bundled actin networks. Science 304: 1301-1305.

Georges PC, Janmey PA (2005) Cell type-specific response to growth on soft materials. J Appl Physiol 98: 1547-1553.

Gordon WE, Bushnell A (1979) Immunofluorescent and ultrastructural studies of polygonal microfilament networks in respreading non-muscle cells. Exp Cell Res 120: $335-348$.

Guilak F, Tedrow JR, Burgkart R (2000) Viscoelastic properties of the cell nucleus. Biochem BiophysRes Commun 269: 781-786.

Ingber DE (2003) Tensegrity I. Cell structure and hierarchical systems biology. J Cell Sci 116: 1157-1173.

Janmey PA, Euteneuer U, Traub P, Schliwa M (1991) Viscoelastic properties of vimentin compared with other filamentous biopolymer networks. J Cell Biol 113: 155160 .

Kamm RD, McVittie AK, Bathe M (2000) On the role of continuum models in mechanobiology. ASME Int Congr Mech Biol 242: 1-9.

Kong HJ, Polte TR, Alsberg E, Mooney DJ (2005) FRET measurements of cell-traction forces and nano-scale clustering of adhesion ligands varied by substrate stiffness. Proc Natl Acad Sci USA 102: 4300-4305.

Kumar S, Maxwell IZ, Heisterkamp A, Polte TR, Lele TP, Salanga M, Mazur E, Ingber DE (2006) Viscoelastic retraction of single living stress fibers and its impact on cell shape, cytoskeletal organization, and extracellular matrix mechanics. Biophys J 90: 3762-3773.

Lim CT, Zhou EH, Quek ST (2006) Mechanical models for living cells - A review. J Biomech 39: 195-216.

Maguire P, Kilpatrick JI, Kelly G, Prendergast PJ, Campbell VA, O'Connell BC, Jarvis SP (2007) Direct mechanical measurement of geodesic structures in rat mesenchymal stem cells. HFSP J 1: 181-191.
Maniotis AJ, Chen CS, Ingber DE (1997) Demonstration of mechanical connections between integrins cytoskeletal filaments, and nucleoplasm that stabilize nuclear structure. Proc Natl Acad Sci USA 94: 849-854.

Maurin B, Canadas P, Baudriller H, Montcourrier P, Bettache N (2008) Mechanical model of cytoskeleton structuration during cell adhesion and spreading. J Biomech 41: 2036-2041.

McGarry JG, Prendergast PJ (2004) A three-dimensional finite element model of an adherent eukaryotic cell. Eur Cells Mater 7: 27-34.

Nicolas A, Geiger B, Safran SA (2004) Cell mechanosensitivity controls the anisotropy of focal adhesions. Proc Natl Acad Sci USA 101: 12520-12525.

Pedersen JA, Swartz MA (2005) Mechanobiology in the third dimension. Ann Biomed Eng 33: 1469-1490.

Peyton SR, Putnam AJ (2005) Extracellular matrix rigidity governs smooth muscle cell motility in a biphasic fashion. J Cell Physiol 204: 198-209.

Sanger JW, Mittal B, Sanger JM (1984) Analysis of myofibrillar structure and assembly using fluorescently labeled contractile proteins. J Cell Biol 98: 825-833.

Sen S, Engler AJ, Discher DE (2009) Matrix strains induced by cells: Computing how far cells can feel. Cell Mol Bioeng 2: 39-48.

Stamenovic D, Wang N (2000) Invited review: Engineering approaches to cytoskeletal mechanics. J Appl Physiol 89: 2085-2090.

Walcott S, Sun SX (2010) A mechanical model of actin stress fiber formation and substrate elasticity sensing in adherent cells. Proc Natl Acad Sci USA 107: 7757-7762.

Wang N, Stamenovic D (2000) Contribution of intermediate filaments to cell stiffness, stiffening, and growth. Am J Physiol Cell Physiol 279: C188-C194.

Wang N, Naruse K, Stamenovic D, Fredberg JJ, Mijailovich SM, Tolić-Nurrelykke IM, Polte T, Mannix R, Ingber DE (2001) Mechanical behavior in living cells consistent with the tensegrity model. Proc Natl Acad Sci USA 98: 7765-7770.

Wendling S, Oddou C, Isabey D (1999) Stiffening response of a cellular tensegrity model. J Theor Biol 196: 309-325.

Yeung T, Georges PC, Flanagan LA, Marg B, Ortiz M, Funaki M, Zahir N, Ming W, Weaver V, Janmey PA (2005) Effects of substrate stiffness on cell morphology, cytoskeletal structure, and adhesion. Cell Motil Cytoskel 60: 24-34.

Zemel A, Rehfeldt F, Brown AEX, Discher DE, Safran SA (2010) Optimal matrix rigidity for stress-fibre polarization in stem cells. Nat Phys 6: 468-473.

Zhu C, Bao G, Wang N (2000) Cell mechanics: Mechanical response, cell adhesion, and molecular deformation. Ann Rev Biomed Eng 2: 189-226.

\section{Web References}

1. http://pyformex.berlios.de/ [11-06-2011]. 


\section{Discussion with Reviewers}

Reviewer I: I am interested in the difference between the finite element model and the simple model that is highlighted by the authors: namely, that - on a rigid substrate - microtubules are unloaded in the simple model, but are loaded in the FE model. Why do you think this difference arises? Is there a simple intuition for it?

Authors: A tensegrity structure is different from a system of springs (tensed and compressed) in a parallel configuration. Tensegrity structures are stable per se because of a tensional prestress, which allows cables to be tensed and rods to be compressed. In our cell model, with a tensegrity structure pinned to a substrate, the compression of the rods is not equal to the difference between the tension of the cables and the compression of the substrate (as it would be for a parallel configuration). Whatever the substrate stiffness (from no-substrate to rigid substrate), the rods are always subjected to an internal preloading. An exceptional case would occur if all vertices of the tensegrity structure would be pinned to a substrate (the tensional prestress would then no be longer required for stability). In this configuration, a tensegrity structure on a substrate would degenerate into a parallel configuration (the compression of the rods would then be equal to the difference between the tension of the cables and the compression of the substrate). On a rigid substrate the rods' loading state would be constant whatever the cable tension (all rods constrained at both sides by fixed pin joints), and could be compressed, unloaded or even tensed. However, this is unlikely to happen in living cells. Assuming that all cell receptors are connected to a rigid substrate, still there will be microtubules with one side located internally (at the centrosome), thus not connected to the substrate. In general, the cytoskeleton is only partially constrained to the substrate by Focal Adhesions and the internal pretension is needed for the stability. Thus, even on a rigid substrate microtubules are compressed.

Reviewer II: In this investigation, four symmetrically spaced focal adhesion contacts between cell and substrate have been considered. I wonder whether cell response to matrix with different elasticity may be affected by consideration of different numbers and location of focal adhesion sites.

Authors: The choice of using a simple tensegrity model (e.g. equally length struts, symmetrically spaced focal adhesion contacts) corresponds to the aim of the study, which is not to reproduce the process of cell adhesion and spreading but to investigate possible underlying rules of substrate-elasticity sensing. Thus, our results are qualitatively valid for any tensegrity-based cell model if at least two FACs are present (with respect to our model, one single FACs would not be sufficient to provide the cell with the substrate-elasticity sensing capability). As a proof, we have repeated the study with a different tensegrity structure (round shape, 6 struts, 24 cables and 3 FACs) and, as expected, we have obtained the same results in terms of sensing the substrate stiffness (sigmoid curve) and cellto-substrate adaptation (SF formation). A comparison of different tensegrity structures (including different contacts between cell and substrate, different cell shape or cell partially embedded into a substrate) is not part of our study and would be interesting.

Reviewer III: Bundling (stiffening) of stress fibres controls and increases the value of the elasticity sensitivity peak (thicker stress fibres for stiffer substrate), while contraction of stress fibres controls the sensitivity magnitude. No results were presented about stress and strain of cytoplasm and nucleus. Are they affected mechanically by matrix elasticity? The nucleus deformation may be a signal of the mechanical state of the cell. How does the nucleus react to changes in stiffness or contraction of stress fibres? Is this signal too low?

Authors: Cytoplasm and nucleus were both under compression and the level of compression depended on the matrix elasticity. The authors believe that, in order to investigate the deformation of the nucleus due to matrix elasticity or contraction of stress fibres, a more realistic model, which would include the heterogeneous sub-nuclear structures, is needed. Hence, we believe that the nucleus model we used (linear elastic, homogeneous, isotropic continuum) is over-simplified and is not suitable to investigate the signalling role of the nucleus.

Reviewer III: Fibroblasts cultured on free-floating collagen gels make many, many actin stress fibres. Indeed, free-floating gels are contracted spectacularly by the fibroblasts in an actin stress fibre dependent manner.

Authors: We acknowledge the widely known behaviour that fibroblasts can contract free floating (unconstrained) collagen gels mentioned by the reviewer. However, the point we were attempting to make is that the morphology and cytoskeletal structure is altered for cells cultured on unconstrained gels. Specifically, our literature review indicated that the mechanism of contraction differs for fibroblasts cultured on unconstrained collagen gels vs. constrained collagen gels. For example, Grinell (2000, 2003, 2008; additional references) has indicated that cells on unconstrained gels tend to have little or no stress fibres and extend fine protrusions into the matrix during contraction. In contrast, cells on constrained gels tend to form large extensions and clearly defined stress fibres leading to isometric tension in the cell and matrix contraction. Evidence of decreased stress fibre generation in cells on unconstrained gels has also been noted in other studies (Hay, 1982; Tomasek et al., 1982; Halliday and Tomasek, 1995; additional references). Hay (1982) and Tomasek et al. (1982) found that fibroblasts on unconstrained gels did not exhibit actin-myosin stress fibres capable of contracting the cell, but instead typically exhibited actin in their cortex and filipodia while myosin resided primarily in their cytosol; thus, they proposed that 
motility of cells cultured on unconstrained gels may result from sliding of cortical actin past myosin in the cytosol rather than from stress-fibre dependent contraction. Grinell (2000) has noted that the precise process of contraction for unconstrained gels is not clear but reinforces the ideas of Hay (1982) and Tomasek et al. (1982) by noting that floating matrix contraction depends on signalling pathways that are important for cell motility. Halliday and Tomasek (1995) and Georges and Janmey (2005) (text reference) have also shown clear examples of rounded fibroblasts on soft gels with no articulated stress fibres (i.e., diffuse actin structure) and fibroblasts with significant extensions and clearly visible stress fibres. However, Halliday and Tomasek (1995) did find flattened fibroblasts with clearly defined stress fibres at the surface of the unconstrained gel.

In conclusion, while we accept the assertion that fibroblasts can contract free-floating collagen gels we have not been able to confirm that fibroblasts form significant stress fibres on free-floating gels with the exception of the gel surface. Therefore, it does not seem clear that matrix contraction in such circumstances is stress-fibre dependent.

\section{Additional References}

Grinnell F (2000) Fibroblast-collagen-matrix contraction: growth-factor signalling and mechanical loading. Trends Cell Biol 10: 362-365.

Grinnell F (2003) Fibroblast biology in threedimensional collagen matrices. Trends Cell Biol 13: $264-$ 269.

Grinnell F (2008) Fibroblast mechanics in threedimensional collagen matrices. J Bodyw Mov Ther 12: 191-193.

Halliday, NL, Tomasek JJ (1995) Mechanical properties of the extracellular matrix influence fibronectin fibril assembly in vitro. Exp. Cell Res 217: 109-117.

Hay ED (1982) Interaction of embryonic surface and cytoskeleton with extracellular matrix. Am J Anat 165: $1-12$.

Tomasek JJ, Hay ED, Fujiwara K (1982) Collagen modulates cell shape and cytoskeleton of embryonic corneal and fibroma fibroblasts: Distribution of actin, $\alpha$-actinin, and myosin. Dev Biol 92: 107-122. 